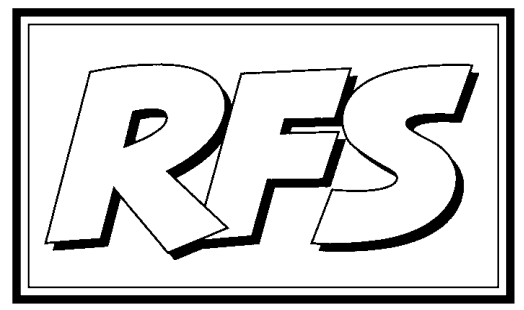

Revista de Fomento Social, 55 (2000), 271-286

\title{
Las migraciones en un mundo globalizado
}

André Linard ${ }^{1}$

Aun cuando este fenómeno ha existido desde siempre, los movimientos migratorios de los últimos años presentan nuevas características. Los emigrantes modernos difieren ligeramente de sus predecesores ya instalados en los países de acogida, aunque éstos en muchos aspectos siguen siendo inmigrantes, con los problemas específicos que se les plantean.

\section{El nuevo rostro de los emigrantes}

En primer lugar, existe un número cada vez mayor de emigrantes. De 1970 a 1990, 6 millones de personas se desplazaron anualmente, con un repunte de 14,5 millones en 1989. Según la División de la población mundial del Departamento de Información Económica y Social de las Naciones Unidas, en la

1 Director de información de la Confederación Mundial del Trabajo (CMT) - Bruselas; profesor en el Institut Catholique des Hautes Etudes Commerciales (ICHEC)- Bruselas. CMT: 33, rue de Trèves, 1040 Bruxelles, Bélgica ICHEC: 2, Boulevard Brand Whitlock, 1150 Bruxelles, Bélgica. 
actualidad existen aproximadamente 130 millones de inmigrantes (hombres y mujeres), de los cuales unos 15 millones son refugiados registrados por el ACNUR.

Sin embargo, es difícil determinar cifras fiables, en primer lugar, por la existencia de flujos clandestinos y, en segundo lugar, por la dificultad de definir al migrante.

La Organización Internacional del Trabajo presenta cifras de entre 42 millones en 1995 (interpretación restrictiva, véase tabla a continuación) y 70 millones de personas que trabajarían legalmente en otro país -cifras a las cuales conviene agregar a los familiares-. En sus estimaciones, la OIT no tiene en cuenta otro fenómeno masivo: las migraciones internas, principalmente en los grandes países (China, Brasil, etc.).

La segunda diferencia se sitúa al nivel de la evolución geográfica. Lejos estamos de la época en que las migraciones tenían su origen en Europa y - ¿casualidad?- no eran consideradas como un problema. Al comparar la situación de hoy con la de hace sólo veinte o treinta años, salta a la vista que los países de origen y destino han cambiado, es decir, durante las últimas décadas, África, el mundo árabe y sobre todo Asia se convirtieron en los principales puntos de partida de los emigrantes, mientras que los países tradicionales de emigración, a excepción de México que sigue enviando 350.000 ciudadanos anuales a EE.UU, pasaron a un segundo plano. Los países de Europa del Este, en donde se estableció la libre circulación de personas, se convirtieron en los lugares de recepción y tránsito, aun cuando en el pasado eran los puntos de partida (al igual que España). Según la Organización de Cooperación y Desarrollo Económicos, "en algunos $\mathrm{PECO}^{2}$ y en Rusia, los flujos de inmigración probablemente son superiores a los flujos de emigración" 3 .

Países de Europa central y oriental.

OCDE, Tendencias..., 1998, p. 51. 


\section{Número de extranjeros legales o no (sin tener en cuenta refugiados y solicitantes de asilo) por continente (en millones - 1995)}

\begin{tabular}{|l|c|c|c|}
\hline Continente & Económicamente Activos & Fa milia res & Tota I \\
\hline África & $6-7$ & $12-14$ & $18-21$ \\
\hline América del Norte & 8 & $8-10$ & $16-18$ \\
\hline América Central y del Sur & $3-5$ & $4-7$ & $7-12$ \\
\hline Asia del Sur, Este y Sudeste & $2-3$ & $3-4$ & $5-7$ \\
\hline Oriente Próximo & 6 & $2-3$ & $8-9$ \\
\hline Europa & $11-13$ & $15-17$ & $26-30$ \\
\hline TOTAL & $26-42$ & $44-45$ & $80-97$ \\
\hline
\end{tabular}

Fuente: OIT: Trabajadores emigrantes, Informe para la Conferencia internacional del Trabajo, Ginebra, 1999, p. 4.

La mayoría de los desplazamientos se realizan ahora entre los países del sur, de donde provienen entre el 70 y el $80 \%$ de los inmigrantes. Asia es el continente que ha conocido el mayor crecimiento de migración (aunque otros países tales como los del Mercosur o del África austral también se ven afectados). En 1995 se estimaba en seis millones el número de emigrantes inter-asiáticos. En 1997, había un total de 4,5 millones de inmigrantes sólo en Tailandia y en Malasia (antes de la crisis). A la inversa, serían más de 6 millones los filipinos emigrantes -se ha llegado a denominar a Filipinas el Reino de la emigración-, 2,5 millones los indonesios emigrados, 1 millón de personas emigradas de Sri Lanka, igual número de nacionales de Bangladesh, etc. Los inmigrados asiáticos son numerosos también en países como Estados Unidos, Canadá, Australia y Japón.

\subsection{Volátiles e impredecibles}

Una tercera novedad es la dispersión. Los movimientos migratorios actuales son más dispersos y diversificados; su flujo es mucho menos estructurado y organizado; y las políticas públicas y las instancias de control de las migraciones parecen no soportar el peso de la realidad. Simultáneamente a la volatilidad del capital, que cruza las fronteras sin mayor control, "los flujos de migración internacional no sólo aumentaron cuantitativamente, sino también fortalecieron su carácter volátil e impredecible", afirma un 
documento de trabajo del Banco Mundial ${ }^{4}$.

También es muy claro el primer párrafo del informe antes mencionado de Ia OCDE: "El análisis de las recientes tendencias de los movimientos migratorios confirma que, en los cuatro últimos años, han disminuido los flujos regulares de inmigración registrados en la mayoría de los países miembros de la OCDE. (...) Las migraciones temporales de mano de obra van en aumento, en particular las que atañen a trabajadores cualificados. Sin embargo, la persistencia de las migraciones irregulares es un claro indicio de las dificultades que enfrentan los países de destino y de origen para controlar estos flujos." ${ }^{5}$

Si éste es el caso en los países de la OCDE, en los que las infraestructuras de control están muy desarrollados y las políticas recientes son más restrictivas, lo es más aún en los países del sur.

Esta evolución esconde otra: la privatización de la migración. Hace unos 30 años, los flujos de mano de obra eran manejados principalmente por las autoridades. Si faltaba mano de obra para trabajar en las minas, por ejemplo, un Estado buscaba concluir un acuerdo de "abastecimiento de mano de obra", en cantidades relativamente precisas.

Estas prácticas no han desaparecido del todo. En la China, por ejemplo, el gobierno motiva y ayuda a sus recién graduados universitarios a emigrar; a la inversa, Canadá organiza la llegada de inmigrantes con calificaciones precisas. Con todo, sin embargo, esta gestión pública dela migración está cediendo poco a poco frente a una organización privatizada, dominada por "contrabandistas" que a veces se convierten en negreros e incurren en una dolorosa explotación de los candidatos emigrantes. "En algunos países de origen, la principal institución interesada -el Estado- se limita a prescribir determinadas formalidades, como en Bangladesh, y deja que agentes privados, amigos o parientes se encarguen de organizar el encuentro entre empleadores extranjeros y candidatos locales." ${ }^{\prime}$ En muchos países, el Estado ni siquiera interviene.

Esta privatización constituye una tendencia general que no sólo aumenta el número de emigrantes, sino que también abre la puerta a muchos vicios: extorsión de fondos, abandono de los clandestinos a medio camino, etc. En 1997, la OIT modificó el Convenio n 96, de 1949, referente a las agencias privadas de empleo, con el fin de incluir a los "contrabandistas". El nuevo

\footnotetext{
S.S. Russel y M.S. Teitelbaum, 1992, p. 7.

OCDE, op. cit., p. 13

W.R. BöHNING, 1999, p. 6.
} 
Convenio obliga a los Estados a proteger a los inmigrantes traídos por estos agentes privados y propone a estos últimos un código de conducta. Cabe preguntarse ahora si el nuevo Convenio será aplicado a los que actúan en la ilegalidad.

\subsection{Más mujeres autónomas}

Otra tendencia manifiesta que se puede observar es la feminización de la migración. Durante mucho tiempo, la mujer sólo se tenía en cuenta en el marco de la reagrupación familiar o era víctima de tráficos que la conducían a la prostitución. ${ }^{7}$ Hoy en día, las mujeres representan una proporción creciente de trabajadores emigrantes "normales", es decir, de personas que toman la decisión de ir a buscar trabajo a otro país; además, a menudo se van solas. De hecho, en el Medio Oriente, habría medio millón de mujeres procedentes de Sri Lanka; de los emigrantes filipinos en Asia, habría doce mujeres por cada hombre.

Un último aspecto nuevo en las migraciones actuales: el aumento de la tasa de trabajadores nada o poco cualificados. En un estudio realizado en 1993 en la India, Sri Lanka y Bangladesh, se ha demostrado que la gran mayoría de los candidatos emigrantes vienen de zonas rurales, son pobres y tienen poca o ninguna instrucción (el $92 \%$ en la India), y además, son mujeres ${ }^{8}$.

"Los trabajadores no cualificados, a menudo sin grado alguno de instrucción (...) representan el grupo cuantitativamente más importante..." ${ }^{\prime 9}$ de los emigrantes. Simultáneamente, los países de acogida procuran reservar la entrada a los trabajadores cualificados que necesitan. Este fenómeno no es contrario al proceso de descualificación que atraviesan muchos trabajadores inmigrantes.

Además, existe un vínculo entre los diferentes aspectos de esta evolución. Los países de destino tradicionales implantaron políticas restrictivas de inmigración regular, destinadas sobre todo a trabajadores cualificados admitidos temporalmente para compensar los déficits de cualificación del país. Por lo tanto, los emigrantes poco o no cualificados se ven obligados a recurrir a redes ilegales de migración establecidas por agentes privados.

\footnotetext{
OIT: Trabajadores..., 1999, p. 11-12.

Movimiento Obrero Cristiano, 1993.

Pierre George, 1985, vol 14, pp. 1059-1060.
} 


\subsection{Los empleos más vulnerables}

Estas diversas facetas de la evolución de las migraciones contribuyen a reforzar la fragilidad de los inmigrantes con respecto a las personas encargadas de su transporte y a sus empleadores, y los hacen más vulnerables a las fluctuaciones económicas. Como son clandestinos, estos inmigrantes no pueden defenderse por el miedo a ser expulsados. Como son poco cualificados, se ven obligados a realizar trabajos de segunda clase.

En los países tradicionales de inmigración (Europa, Norteamérica), tiende a disminuir el empleo industrial de poca cualificación, a beneficio del empleo de mayor cualificación o del empleo en el sector terciario. Esta evolución afecta más duramente a los trabajadores inmigrados, generalmente menos cualificados. De hecho, según la OCDE, la mano de obra masculina extranjera se concentra en los sectores en declive o en reestructuración (industria), así como en los sectores más expuestos a las fluctuaciones coyunturales (construcción).

\footnotetext{
"Para el conjunto de la mano de obra, las pérdidas de empleo en la industria han sido compensadas por empleos generados en el sector terciario. El empleo extranjero ha tenido una evolución similar, pero los empleos creados en el sector terciario no han podido compensar las pérdidas masivas en la industria, en particular en Bélgica, Francia y Alemania"10.
}

La principal consecuencia es que los inmigrantes son más vulnerables al desempleo que los nativos del país, lo que se ve reflejado en un desempleo más elevado entre los extranjeros, tal como se ha observado en muchos países de la OCDE. De ahí que los extranjeros se dedican a trabajos no asalariados que, como se sabe, presentan un aspecto "noble" (condición de trabajadores independientes) y otro más polémico (trabajo no declarado, tráfico...). Pero en ambos casos, la protección social de estos trabajadores es generalmente menor.

\section{2. ¿Por qué marcharse? Entre la demanda y la oferta}

Es muy común explicar el fenómeno de las migraciones a través de las razones que llevan al emigrante a abandonar su país para probar suerte en el extranjero. El geógrafo Pierre George, por ejemplo, explica: “las migraciones económicas son generalmente provocadas por el deterioro del nivel de vida

10 OCDE, op. cit., p. 39. 
de poblaciones cuyo crecimiento demográfico no se ha visto correspondido por un suficiente aumento de los recursos; también suelen afectar más específicamente a las clases menos favorecidas" ${ }^{11}$. Este enfoque considera que en la decisión del emigrante es determinante la situación de su país y contempla la emigración como una estrategia para responder a esta situación. Ciertamente estos factores entran en juego, y serán estudiados más adelante, pero no son los únicos.

Otro punto de partida para estudiar la emigración es la existencia de una demanda de mano de obra en los países de destino. En el pasado, y aún hoy en día en ciertos casos (sureste asiático hasta mediados de 1997, por ejemplo), esta demanda era el resultado de una desproporción matemática entre el número de empleos ofrecidos y la mano de obra disponible. Sin embargo, en la mayoría de los casos, el análisis debe ser profundizado y tener en cuenta dos factores: por una parte, el hecho de que una parte de la mano de obra del país de destino se resista a desempeñar ciertos trabajos mal remunerados o realizados en condiciones deplorables, y por otra parte el hecho de que los empleadores buscan una mano de obra lo más económica posible, tanto en términos de salarios directos como en otros costos.

En Malasia, por ejemplo, el sector de la construcción depende en un 80\% de la mano de obra extranjera, empleada en los trabajos menos remunerados y desconsiderados por los malayos. En lugar de mejorar las condiciones de trabajo, los empleadores prefieren importar una mano de obra que, por ser más pobre, tiende menos a exigir reivindicaciones.

\subsection{La demanda: un conflicto de intereses}

En realidad, los países de destino tienen varios mercados laborales yuxtapuestos, divididos según el nivel salarial, las calificaciones requeridas y las condiciones de trabajo. En los mercados más desfavorables para los trabajadores, la oferta local de mano de obra suele ser insuficiente. Por esta razón, desde el punto de vista económico, los inmigrantes responden a una necesidad de mano de obra, perfectamente compatible con una alta tasa de desempleo en la economía.

Pero este argumento económico puede enfrentarse a un rechazo más o menos agudo del extranjero por parte de la opinión pública (cfr. el ejemplo

11 Pierre George, op. cit. 
de El Ejido en febrero 2000) y, a veces, por parte de los políticos.

Parecen actuar simultáneamente dos lógicas, pero con una tensión entre sí: la lógica del costo de la mano de obra en una economía capitalista, que incita a la contratación de inmigrantes menos costosos y menos exigentes, y la lógica política de la identidad nacional que les niega la ciudadanía a los extranjeros. Es también la lógica de la racionalidad económica neoliberal, que exige una mano de obra barata, frentea la lógica de la psicología colectiva que encuentra un chivo expiatorio en el inmigrado.

Esta quizás sea la explicación de la decisión, por parte de Hong Kong, en plena crisis asiática y mientras los demás países de la zona expulsaban a sus trabajadores inmigrados, de "importar" nuevos trabajadores extranjeros para el sector de la construcción, en contra, además, de la opinión de los sindicatos locales que se hacían eco del temor de sus miembros frente a la competencia de los trabajadores inmigrantes.

Este conflicto de interés se resuelve en parte privando al inmigrado de la ciudadanía. "La llegada de nuevas poblaciones en un territorio delimitado por un Estado-Nación se realiza en primer lugar ( ...) a través de un ingreso salarial discriminado, asociado eventualmente con una función demográfica. Esta concepción de las migraciones modernas (...) se codificó en el Tratado de Roma. En efecto, en este texto se conceptualizan las migraciones como 'la libre circulación de la mano de obra', un concepto definido dentro de una 'política de empleo'" afirman Albert Bastenier y Felice Dassetto. ${ }^{12}$

Aun cuando el emigrante ya se haya instalado en el país de destino, continúa siendo un ciudadano de segunda clase, generalmente privado del derecho al voto. El emigrante es aceptado con la condición de seguir siendo diferente: "el inmigrado es un interesante asalariado, precisamente porque ocupa una posición social marginal"13, escriben los mismos. Se aceptan los brazos para trabajar, pero no los hombres ni las mujeres a quienes pertenecen. No sólo es económico el problema, sino también político y ético.

\subsection{La oferta: el modelo de Todaro}

Todos los factores de producción son objetos de una oferta y una demanda por encima de las fronteras. La mano de obra también. La migración es el

12 Albert Bastenier y Felice Dassetto, 1990, p. 12. 
resultado de la confluencia entre la demanda de mano de obra y una oferta. De hecho, millones de candidatos están dispuestos a emigrar, motivados, en el caso de trabajadores, por su afán por conseguir empleo e ingresos.

Michael Todaro, economista, intentó formalizar la motivación de aquellos queemigran de las zonas rurales hacia las ciudades y sus industrias. El mismo razonamiento podría aplicarse a las migraciones internacionales. Todaro afirma que los trabajadores deciden abandonar o no su entorno de origen de acuerdo con los costos y las ventajas que sacan o esperan sacar de cada situación, incluyendo así un cálculo de probabilidades ${ }^{14}$.

En la decisión influyen las ventajas inherentes al entorno original (seguridad del ingreso, aun bajo, estabilidad, etc.) y los inconvenientes que implica el marcharse (incertidumbre, riesgo de perderlo todo, ruptura cultural y familiar, costo del viaje, etc.). Pero también entran en juego los inconvenientes del entorno original (ingreso inferior y a menudo insuficiente, el aislamiento con relación al "mundo moderno", etc.) y las ventajas de la emigración (esperanza de tener mayores ingresos y una vida mejor, etc.).

Se produce la emigración cuando la ganancia incierta pero esperada, moderada por sus costos, es superior a la ganancia obtenida con toda seguridad en el lugar de origen.

Los factores clave son, pues, la enorme brecha entre el ingreso obtenido en el lugar de origen y el esperado en el país de destino; la probabilidad de conseguir un empleo (que depende de la demanda de mano de obra en el país de destino); y la información que tenga el emigrante potencial sobre estos elementos.

El candidato a emigrante no se desanimará por el hecho de vivir en condiciones menos favorables que el ciudadano del país de destino. Lo que importa, para él, es la diferencia entre lo que posee y lo que espera ganar. Además, cada nueva oferta de trabajo en los países de destino genera la emigración de varios candidatos. El modelo de Todaro, que remite a un cálculo racional de los costos y beneficios realizado por el emigrante potencial, ignora en efecto un factor subjetivo: el hecho de que aun cuando la probabilidad de éxito es muy reducida, el emigrante está convencido de que él sí lo va a lograr, gracias a su coraje, su dedicación al trabajo, etc. Es así

13 Ibid., p. 15.

14 Michael Todaro y John R. Harris, 1970. 
como se producen migraciones espontáneas hacia países con una mano de obra excedentaria, producto de la convicción de los inmigrantes de que "siempre habrá manera de conseguir un trabajo", reforzada, además, por la imagen de éxito, verdadera o falsa, dada por los inmigrantes anteriores.

Por lo tanto, las migraciones tienen un futuro prometedor, ya que las brechas de riqueza no paran de aumentar, no sólo entre el Norte y el Sur, sino también entre las regiones del Sur y dentro de la mayoría de los países. Desde 1965, el porcentaje de ingresos en el ámbito mundial correspondiente al $20 \%$ de los más pobres cayó de $2,3 \%$ a $1,4 \%$, mientras que el del $20 \%$ de los más ricos pasó de $70 \%$ a $85 \%$. La brecha de ingresos entre el $20 \%$ más pobre y el $20 \%$ más rico se duplicó y pasó de 30 por 1 , a 74 por $1^{15}$, y nada indica que este proceso pueda revertirse.

\section{La globalización incrementa las migraciones}

Cuando se habla de globalización, se suele pensar en los capitales golondrinas, en el debilitamiento del poder público, en la concentración del poder de decisión en unas grandes corporaciones financieras y en la libre circulación de las empresas, servicios, bienes y factores de producción. Sin embargo, esta última noción también incluye otro elemento, poco mencionado en estas discusiones: el trabajo. En el diario francés Le Monde, Claude Pottier escribía: "a menudo se reduce la mundialización a su dimensión comercial, pero la nueva realidad no resulta tanto de la intensificación del comercio como de una mayor movilidad internacional de los factores de producción"16.

El Grupo de Lisboa, animado entre otros por Riccardo Petrella, propone las claves para comprender cómo la mundialización influye en las migraciones, distinguiendo tres etapas: internacionalización, multinacionalización y globalización ${ }^{17}$. La primera se refleja en la intensidad del comercio exterior y de los movimientos poblacionales, para cuyo monitoreo y orientación las autoridades públicas disponen de las estadísticas. En un contexto de internacionalización, las autoridades tienen "el control de los movimientos poblacionales y regulan los temas relacionados con la ciudadanía, a través de la apertura 0 el cierre de las fronteras". La multinacionalización se caracteriza

\footnotetext{
15 PNUD, 1999.

16 Le Monde, 4 de noviembre de 1977.

17 Grupo de Lisboa (coordinación de Riccardo Petrella), 1994.
} 
por la deslocalización de los recursos, en primer lugar del capital y, en menor medida, dela mano de obra. En esta etapa las fronteras nacionales pueden ser traspasadas cada vez con más facilidad.

Finalmente, la etapa de globalización marca el debilitamiento del poder de las autoridades públicas nacionales. "Es difícil atribuir un territorio en particular (desde el punto de vista jurídico, económico, tecnológico u otro) a una organización [ presente en el mundo entero], aun cuando tenga una patria de origen...". Las tres palabras clave de esta mundialización son: liberalización, privatización y desregulación, inclusive para los movimientos poblacionales.

\subsection{Migraciones y comercio}

Los economistas no están de acuerdo en torno a la influencia del crecimiento de las relaciones comerciales internacionales sobre los flujos migratorios. Algunos opinan que este crecimiento desalienta las migraciones, ya que transfiriendo la producción hacia los países de origen de los inmigrantes, se reducen los desplazamientos de mano de obra. Un informe de la OCDE adopta esta concepción y aprovecha para exhortar a los países en desarrollo a que abran sus fronteras a las inversiones extranjeras y liberalicen sus intercambios comerciales para reducir la emigración ${ }^{18}$. Concluye la OCDE diciendo que "la incitación a emigrar irá disminuyendo gracias a un impulso duradero acompañado de la creación de empleos".

Sin embargo, esta tesis tiene argumentos en contra. Si bien el TLCAN ${ }^{19}$ ha intensificado las inversiones de empresas estadounidenses en México, no ha logrado desmotivar la emigración de los Mexicanos hacia el norte, sobre todo por las diferencias salariales. Lo que se requiere, pues, es una política voluntarista y eficiente por parte de los países de destino, para limitar la inmigración ilegal, suprimir las llegadas ilegales de inmigrantes e inclusive apoyar las inversiones directas de sus empresas nacionales en los países de origen ${ }^{20}$. Pero estas condiciones son difíciles de cumplir.

La tesis explicada anteriormente también omite el hecho de que muchas de las tareas realizadas por los inmigrados no pueden deslocalizarse por ser

18 OCDE, Tendencias..., 1998,

19 Tratado de Libre Comercio del Atlántico Norte.

20 MAURICE SHIFF, 1996. 
tareas de proximidad (limpieza, transporte público, servicios a las personas, etc.) y las empresas de estos sectores siguen necesitando una mano de obra barata. Finalmente esta tesis no tiene en cuenta el hecho de que los niveles actuales de ayuda al desarrollo son muy inferiores a las remesas en divisas que envían los emigrados a sus países de origen.

Por estas razones, es más correcto contemplar la apertura al comercio y a los capitales extranjeros y la intensificación de las migraciones como complementarias. Dentro de la Unión Europea, donde está vigente la libre circulación de las personas, ha pasado a ser común el tener un empleo en un país miembro diferente al de nacimiento. Cuando la integración económica pone en relación países con niveles desiguales de desarrollo, la migración se intensifica. Además, los medios modernos de información y comunicación permiten conocer las realidades de otros países y facilitan la ruptura con la tradición del entorno original. "Lo otro", lo ajeno, ya no da tanto miedo y poco importa que el conocimiento de otra realidad sea correcto o no. Al contrario: mientras más ilusoria sea la imagen del país de destino, más será atractiva, en la medida en que valorizará los estilos "modernos" de vida y la posibilidad de éxito social. Y como, de paso, los medios de transporte también van perfeccionándose, la movilidad de las personas se ve aún más facilitada.

\section{Un desafío para los sindicatos}

Las migraciones interpelan al movimiento sindical en los niveles mundial, continental y nacional, porque dibujan el panorama del mundo del trabajo.

En términos generales, los sindicatos pueden enorgullecerse de haber logrado la integración de los trabajadores inmigrantes a las elecciones sindicales, con derecho a ser tanto elector como candidato, mucho antes de que los Estados los convirtiera en ciudadanos. Pero no todo es color de rosa en este aspecto de la vida sindical. Subjetiva y objetivamente, se puede entender que algunos consideren la inmigración como una amenaza.

Desde el punto subjetivo, en primer lugar. Como los inmigrantes tienen menos cualificación y trabajan en los sectores que se encuentran en plena reestructuración, sus colegas nacionales los pueden percibir como competidores directos y, por ende, como una amenaza para sus propios puestos de trabajo. De hecho, como escribe Claude Pottier, "la mundialización pone a los asalariados del mundo entero en una situación de competencia directa, con la subsecuente tendencia a la baja de las condiciones de trabajo y de 
remuneración"21. No se tiene que buscar más lejos la alta proporción de votos de extrema-derecha en periferias obreras de grandes ciudades. El tradicional lema "divide y vencerás" fue substituido por "haz que se abran las fronteras y vencerás". En respuesta a las reacciones de algunos de sus miembros, los sindicatos corren el riesgo de adoptar posiciones xenófobas.

Además, de manera objetiva, en una economía neoliberal basada en el "todo mercado", el trabajo se convierte en una mera mercancía sujeta a la ley de la oferta y la demanda. Cuando hay competencia entre trabajadores por una excesiva oferta con relación a la demanda, el costo de la mano de obra tiende a bajar, lo que lleva a Claude Pottier a añadir que "en los países industrializados, la inmigración debilita el poder de negociación de los asalariados frente a sus empleadores".

Pero no hay que equivocarse de adversario: los trabajadores inmigrantes son las víctimas del sistema, no los responsables. Combinada con la pérdida de poder de los Estados nacionales, la migración descompartimentaliza los mercados laborales nacionales, creando un mercado laboral mundializado. Pero históricamente, las conquistas sociales de los trabajadores se lograron en los marcos nacionales, con base en luchas y relaciones de fuerzas particulares. La mundialización obliga a la transposición de estas relaciones de fuerzas hacia el plano mundial, en el que dominan los agentes empresarios, pese a los esfuerzos ya adelantados por las organizaciones sindicales internacionales o continentales. En la situación actual, el incremento de los flujos migratorios puede volcarse en contra de los trabajadores.

Esta es una razón más para que se busque definir una reglamentación internacional del trabajo, que tenga eficacia para los trabajadores en general y para los emigrantes en particular. Textos de este tipo ya existen, en la OIT 0 en las Naciones Unidas; se trata ahora de lograr que se apliquen. Otros instrumentos quizás deban ser elaborados ${ }^{22}$.

21 Le Monde, 4 de noviembre de 1977.

22 En 1998, la CMT realizó un seminario sobre el papel de los sindicatos en la protección de los derechos de los trabajadores emigrantes. Los documentos de este evento fueron publicados con el título Seminario de la CMT sobre los emigrantes. Ver también: CMT: Informe anual sobre los derechos de los trabajadores. 1999: Trabajadores emigrantes, $48 \mathrm{p}$. 


\section{Conclusión ¿Orden o desorden?}

Las personas que emigran de un país a otro en búsqueda de empleos y recursos cambian de perfil, cualitativamente, y son cada vez más numerosas, menos cualificadas, más femeninas. Sus desplazamientos se hacen de manera más dispersa y menos controlable.

Este nuevo escenario de las migraciones internacionales puede ser interpretado como desorden: los emigrantes vendrían a perturbar un sistema estable de Estados y naciones donde cada cual se queda donde está y con quien está. Esta es la percepción de aquellos que se sienten, por razones justificadas o no, amenazados en su propio lugar de trabajo por los extranjeros.

Los movimientos migratorios "no son otra cosa que el reflejo de un mundo dominado por la precariedad del empleo y por los cambios rápidos en la organización de la producción". En otras palabras, "¿no serían más bien el reflejo de un 'nuevo desorden internacional'?", pregunta Pierre Guengant ${ }^{23}$. Si es así, se necesita un reordenamiento: las migraciones tenderían que disminuir y los emigrantes deberían regresar a sus hogares.

Pero también pueden interpretarse estos movimientos migratorios como un elemento más del nuevo orden económico, globalizado, que se está implantando y en el cual los flujos migratorios se vuelven tan comunes como hoy en día la transferencia de capitales. Entonces, Ias migraciones serían, según las palabras de Pierre Guengant "el equivalente humano de la circulación de bienes y capitales, y la flexibilidad de la mano de obra se convertiría en una regla en todos los países".

La segunda hipótesis es más real: "La brecha económica y demográfica creciente que separa a los países industrializados de los países en desarrollo no hará más que reforzar el fenómeno de las migraciones. Por lo tanto, dicho fenómeno representa un componente estructural de la economía mundial contemporánea." 24

En efecto, todo indica que a pesar de las reacciones xenófobas que producen, las migraciones representarán una realidad ineludible en el futuro, tal como lo fueron en el pasado. El profesor Appelyard, editor de la revista International Migrations, opina, al igual que muchos, que el fenómeno migra-

23 Pierre Guingant, 1996.

24 Comisión francesa de Justicia y Paz, Les 100 mots du développement, La Découverte, 1990. 
torio no dejará de crecer, sobre todo entre los países del Sur ${ }^{25}$. En el ámbito político, lo importante no es encontrar la forma de frenar las migraciones, y mucho menos de enviar a los inmigrantes a sus países. El reto consiste sobre todo en encontrar los métodos más eficaces para respetar y proteger a los hombres y a las mujeres, seres humanos y plenos trabajadores, quienes tienen el valor de renunciar a todo para emprender una nueva vida y cuyo trabajo a menudo ha contribuido en gran medida al bienestar del que gozan los nativos del país de destino.

Finalmente, en las mentes de las personas, el reto consiste en reconocer en la presencia de los inmigrantes una oportunidad y no una amenaza y eliminar el sentimiento de competencia.

De todas formas, la historia de la humanidad se ha ido tejiendo de migraciones y mestizaje. La mayoría de los latino-americanos tienen raíces asiáticas y europeas. Los franceses aparentemente serían ingleses que pasaron por España durante las glaciaciones antes de volver a sus tierras y, para al gunos, antes de pararseen el camino. ¿Quién, ahora, puede reivindicar sin reír una «pureza de sangre»? No hay razón por la cual esta historia tendría que detenerse.

\section{Bibliografía}

Bastenier, A. y Dassetto F. (1990), Immigrations et nouveaux pluralismes, De Boeck Université, Louvain-la-Neuve.

BöHNING, W.R. (1999), The intra-Asian migration of workers with special reference to women, Brotherhood of Asian Trade Unionists (BATU), Pasay City, marzo.

Confederación Mundial del Trabajo: Informe anual sobre los derechos de los trabajadores 1999: Trabajadores emigrantes, 48 pág.

George, P. (1985), Enciclopedia Universalis, Vº, Poblaciones (Geografía de las), vol. 14.

Grupo de LISBOA, (coordinación de Riccardo Petrella), Los límites de la competitividad, Bruselas, Ed. Labor; existe en francés, portugués, italiano, holandés, español, japonés, ruso, etc.

25 International Migrations, XXXIII/3-4, 1995. 
Guingant, P. (1996), «Migrations internationales et développement: les nouveaux paradigmes », Revue européenne des migrations internationales, Poitiers, vol. 212.

Movimiento Obrero Cristiano, (1993), Challenge of the times: challenge to join hands in solidarity to liberate the migrant workers in South Asian region, Bruselas, Solidaridad Mundial.

Ocde (Organización de Cooperación y Desarrollo Económico): (1998), Tendencias de las migraciones internacionales. Informe Anual.

Oit (Oficina Internacional del Trabajo): (1999), Trabajadores emigrantes, Informe III para la $87^{\text {a }}$ Conferencia Internacional del Trabajo, Ginebra.

Pnud (Programa de las Naciones Unidas para el Desarrollo): Informe Mundial sobre el Desarrollo Humano 1999.

Russel, S.S. y Teitelbaum, M.S. (1992), International Migrations and International Trade, Documento de Trabajo del Banco Mundial $n^{\circ} 160$.

SHIFF, M. (1996), «Trade Policy and International Migration: Substitutes or Complements?», Development Strategy, Employmentand Migration: insights for models, Ed. J.E. Taylor, OCDE.

TodARo M. y HarRIS J . R. (1970), «Migration, unemployment and development: a two-sector analysis», American Economic Review, 60, marzo, pp. 126142. 


\section{TESTIMONIO: LOS AFRICANOS DE MAGHNIA ${ }^{1}$}

Maghnia es una ciudad fronteriza argelina situada al otro lado de la marroquí Oujda. En los alrededores de la frontera se amontonan en condiciones infrahumanas los inmigrantes procedentes del África subsahariana, a la espera de entrar en Marruecos para intentar entrar en España y Europa a través del filtro de los enclaves de Ceuta y Melilla. El autor de este testimonio es un sacerdote jesuita que trabaja en Orán y visita asiduamente este campo de inmigrantes.

¿Qué decir para dar cuenta de esta realidad de la que somos testigos cada vez que nos desplazamos a Maghnia para encontrarnos allí con los africanos que acampan y transitan a lo largo del oued (arroyo) Jorgi? Siempre me parecen muy numerosos, sin duda son por lo menos dos mil, y se refugian en unas especies de chozas de plástico negro que les protegen de la lluvia pero no del frío. Cada semana constatamos algunos cambios: algunos han partido, otros acaban de llegar del sur, otros han regresado, rechazados por Marruecos. Las dificultades son grandes, pero todos estos jóvenes parecen siempre muy motivados para proseguir su aventura y afrontar los riesgos. Viven gracias al dinero que han traído o que han ganado a lo largo de algunas etapas de su itinerario, pero lo largo del viaje les hace agotar con frecuencia todos sus recursos. Una camerunesa me ha demostrado cómo se podía cocinar un "plato suculento" con algunas patas de pollo. Su cacerola estaba llena de ellas. La solidaridad juega un gran papel aquí y todos están invitados a compartir la olla. Desde comienzos de la tarde, se observa cómo se activan los cocineros. La comida tiene lugar habitualmente hacia las 6 de la tarde, antes de la caída de la tarde. Alrededor del fuego, comienzan entonces largas veladas de conversación, de cantos, también de oración. Ya ha pasado la medianoche cuando se separan, cuando la necesidad de dormir es más fuerte que la sensación de frío.

La juventud y la motivación les dan fuerza para soportar una vida tan dura. Pero, cuando falta la salud, to do se viene abajo. Varios de estos emigrantes han muerto durante el invierno, lejos de su familia,

1 Tomado de: Le Lien, Boletín de información de la diócesis de Orán. Febrero 2000, p. 16. 
de su país, lejos de todo. El servicio de medicina legal del hospital hace una encuesta para conocer la identidad de los que han fallecido, pero a veces sin resultado. Es así como algunos deestos africanos han tenid o que ser enterrados de manera totalmente anónima, sin que sea posible tomar contacto con sus familias que, sin duda, creen que siguen vivos.

Cuando nos reunimos con estos africanos en el oued Jorgi, sentimos con mucha fuerza cuán lejos estamos de esa realidad. Perdidos en medio de esta multitud africana, que llega de numerosos países, somos nosotros los que nos sentimos extraños, extranjeros a esta realidad que recorremos al caminar por el lecho del arroyo, en medio de todos estos hombres venidos de otra parte, y en camino hacia otra parte. Afortunadamente, la relación con ellos es fácil, distendida, a veces cordial. Ello queda facilitado por el hecho de que, en cierta manera, nosotros estamos en su casa, y son ellos los que nos reciben y nos acogen. Muchos de ellos, que han llegado aquí después de diversos y fallidos intentos, nos han visto ya, en alguna de las más de cincuenta visitas que les hemos hecho desde hace un año.

Cada semana tomamos la ruta de Maghnia. A veces tenemos que animar una oración o incluso celebrar una misa, para responder a una petición insistente de los cristianos. Con más frecuencia, nuestra visita sólo pretende expresar un encuentro humano, con el sentimiento de que eso tiene mucho valor.

Bernard LAPIZE 